\title{
Systemic Risks and Resilience of Global Financial Networks
}

\author{
Akira Namatame* \\ Department of Computer Sciences \\ National Defense Academy of Japan \\ Yokosuka, Japan \\ nama@nda.ac.jp \\ *Corresponding author
}

\author{
Kiyotaka Ide \\ Department of Computer Sciences \\ National Defense Academy of Japan \\ Yokosuka, Japan \\ $13001 @$ nda.ac.jp
}

\begin{abstract}
The current financial crisis has triggered research interests on financial networks, since the linkages created by liabilities among banks and other financial institutions play a crucial role, but they are poorly understood. Understanding the structures of financial networks holds the key to understanding its function and the investigations of their network properties gain more attention. One remarkable finding is that financial networks in many countries share some apparently universal features. A prominent example is a highly skewed degree distribution with core-periphery structures. In this paper, we try to identify key network properties that influence our two variants of contagious default. We formulate the global financial networks as modular networks and study the resilience of global financial networks from the perspective of systemic risk. We highlight that systemic risks of the global financial networks is mainly determined by the property of the sub-module network which forms the basic unit of the global modular networks.
\end{abstract}

Keywords-component; stemic risk; cascade failure;core-peripher; modular network

\section{INTRODUCTION}

The financial crisis has revealed the danger of systemic risk due to contagion effects given the interconnectedness of modern banking systems. In banking systems, there is a tendency for crises to spread from one institution to another. This tendency is referred to as systemic risk and, at a large enough scale, leads to systemic failure [1]. Most of the current research using network theory focuses on issues such as financial stability and contagion. In an interbank market, banks with liquidity shortages can borrow liquidity from banks with liquidity surpluses. This interconnection of the banking system can lead to an enhanced liquidity allocation, but it also contributes to risk contagion among banks. Interbank linkages in interbank markets might become a contagion channel through which solvency or liquidity problems of a single bank can spread to other banks. Direct interbank connections become a source of systemic risk, which has highlighted the importance of interbank markets for financial stability [2, 3].

The main purpose of the study on systemic risk is to analyze contagious bank defaults, i.e. defaultsnot caused by the fundamental weakness of a given bank but triggered by failures in thebanking network. As failing banks become unable to honor their commitments on the interbankmarket, they may cause other banks to default, which may in turn push even more banks overthe edge in so-called default cascades [4].
Understanding the determinants of contagiousnessis one of the most challenging questions in modeling an interbank network. A systemically important bank could be defined as a bank that adversely affects a number of other banks in case it runs into trouble itself. Identifying systemically important banks has become one of the key objectives of systemic risk assessment. Systemically important banks can be identified in many different ways. Many researches have been done to contribute to this important discussion by applying techniques from network analysis [5]. In general, network analysis requires two input arguments. First, it takes a network, which could either be given or derived through a network formation process. Second, each network analysis needs an objective. We view the interbank lending market as a network where each participating bank is a nodeand each credit is a link.

In recent studies financial networks have stylized features with the presence of a particular type of meso-scale structure known as core-periphery, in which a densely connected subset of nodes (core) coexists with a sparsely connected partition (periphery), while the members of the core act as intermediaries between members of the periphery [6]. The composition of the cores, whose constituents are identified as the set of systemically important international financial centers, is rather small and remains stable over time. Therefore the macroscopic data such as the degree distribution, correlation among nodes are not enough to set up simulation models to systemic risk. Identifying intermediate-scale structures in an endogenous way is an important aspect in the analysis of complex networks [7], as they allow us to discover features that are not evident either at the global level of aggregate statistics or at the local scale of nodes and edges. In particular, the idea is to partition the network of national financial markets into a densely connected core and a sparsely connected periphery, by testing the additional assumption that countries in the core are well-connected to those in the periphery while the opposite does not hold true. The components of the core are thus central to the network, either because they are densely interconnected or because they are on the shortest paths linking other nodes. The identification of such critical players in the international web of exposures has crucial implications for macro-prudential surveillance, and hence for financial stability.

In this paper, we consider the most and least susceptible network topologies against systemic risks based on the threshold-based cascade [8]. Such networks are characterized 
core-periphery structures consists of hub nodes and stub nodes. The hub nodes form a near complete graph, and stub nodes are connected mainly to hub nodes. We design a large-scale financial network as modular networks using the least and most susceptible networks as basic design units. We investigate the resilience of global financial networks from the perspective of systemic risk. We highlight that systemic risks of the global financial networks is mainly determined by the property of the basic design units.

\section{FINANCIAL NETWORKS}

Allen and Gale [9] investigated the resilience of financial networks to shocks and the formation of financial networks. They explored critical issues in the study of systemic risks by exploring fundamental questions such as how resilient financial networks are to contagion and how financial institutions form connections when exposed to the risk of contagion. They studied how the financial system responds to contagion when financial institutions are connected under different network topologies. They also explored several critical issues of the study of systemic risks by studying two questions: how resilient financial networks are to contagion, and how financial institutions form connections when exposed to the risk of contagion. While more links between banks might be expected to increase the risk of contagion, densely connected banking systems may be less susceptible to contagion than those with a sparse network structure with few connections. They also showed that incomplete networks are more prone to contagion than complete structures. For instance, better-connected networks are more resilient, since the proportion of losses in one bank's portfolio is transferred to more banks through interbank agreements. On the other hand, in the case of an incomplete network, the failure of one bank may trigger the failure of the entire network.

Most studies on the topology of interbank markets have been conducted by investigating a network formed by interbank liabilities. Overall, the most important findings of this literature are: (1) interbank networks are sparse, i.e. the density is low, (2) degree distributions appear to be scale-free with coefficients between $2-3$, (3) transaction volumes appear to follow scalefree distributions as well, (4) interbank networks show disassortative, so small banks tend to trade with large banks and vice versa $[10,11]$.

Many authors note that there is some kind of community structure in the network. For example, Soramäki et al. [12] show that the core-periphery network includes a tightly connected core of money-center banks to which all other banks connect. Thus there is some form of tiering in the interbank market.In the study of tiered banking system, Fricke and Lux [13] show that tiered system with money-center banks, where banks on the periphery are linked to the center but not to each other, may also be susceptible to contagion.Teteryatnikova[14] constructs tiered banking networks, where banks are linked by interbank exposures with a certain predefined probability. The tiered structure is represented either by a network with negative correlation in connectivity of neighboring banks, or alternatively, by a network with a scale-free distribution of connectivity across banks.
Identifying core-periphery structure in interbank markets is an important aspect and several authors identify the set of arguably systemically important (core) banks. The coreperiphery model has been applied in a Craig and von Peter [11] is the first and so far only contribution applying the coreperiphery model to the interbank market. Fricke and Lux [13] investigate the Italian interbank market on the e-MID trading platform from January 1999 to December 2010, and they show the core-periphery network structure. They also apply this core-periphery framework to a data set of credit relationships in Italy and between German. Their findings are in line with the results in Fricke and Lux [13], where the Italian interbank market is shown to display a hierarchical core-periphery structure. The set of highly connected core banks tends to lend money to other core banks and a large number of loosely connected periphery banks, which in turn tend to lend money to a small number of selected core banks, but appear to trade relatively scarcely among themselves.

A core-periphery structure of a network is a division of the nodes into a densely connected core and a sparsely connected periphery. The nodes in the core should also be reasonably well-connected to the nodes in the periphery, but nodes in the periphery are not well-connected to the core. Hence, a node belongs to the core if and only if it is well-connected both to other nodes in the core and to nodes assigned to the periphery. A core structure in a network is thus not merely densely connected but also tends to be central to the network in terms of short paths through the network. The latter feature also helps to distinguish core-periphery structure from community structure. Many networks can have a perfect core-periphery structure as well as both core-periphery structure and community structure, so it is desirable to develop measurements that allow one to examine types of coreperiphery structures.

The literature mentioned above mostly investigates how different network structures respond to the breakdown of a single bank in order to identify which ones are more fragile. But we still cannot obtain a clear picture about whether there exists a certain network that can well withstand shocks, that is, the one that has a high stability against shocks. Motivated by these considerations, we construct in this paper a tiered network model and numerically analyze contagion risk on different types of networks and then study how resilient different network models are against shocks.

Tiered structure is detected in a range of countries' interbank markets, such as the Austrian, German and Italy interbank markets and other markets, and is commonly defined as an organization of lending-borrowing relations between banks, where relatively few first-tier or core banks have a large number of interbank linkages, whereas many second-tier or peripheral banks have only few links. First-tier banks are connected to second-tier banks and are also connected with each other, whereas second-tier banks are almost exclusively connected to first-tier banks. Further, some empirical studies find close parallels to scale-free networks in real-world tiered banking systems. 
However, the finding of interbank networks to be scalefree (SF) and disassortative at the same time are hard to combine with the analytical results of Newman [15], showing that SF networks based on the preferential attachment with a typical scaling exponent of $\alpha=3$ are unassortative, i.e. neither assortative nor disassortative. Therefore, the evidence for a SF distribution together with disassortativity motivates investigation of whether SF networks are unassortative in general, and whether the results are different for a finite size of the network. SF random networks with relatively small scaling exponents tend to be disassortative. However, the degree of dissasortativity is still stronger for the observed networks than for SF networks with the scaling exponent of 2.3 reported in the literature. Another finding is that even small changes in the scaling exponent can lead to substantially different mixing patterns. Some studies underscore the importance of considering core-periphery structure. For example, Chung [16] showed that power-law graphs, in which the number of nodes of degree $\mathrm{k}$ is proportional to $\mathrm{k}^{-\beta}, \beta \in(2,3)$, almost surely contain a dense sub-graph that has short distance to almost all other nodes in the graph. This suggests that it is sensible for networks with heavy-tailed degree distributions to contain some sort of cohesive core.

\section{SYSTEMIC RISK}

How do we keep the performance of networked systems under a chain of failures? The networked system hold a certain danger that a failure of only single node in the networked system can wipe out all other nodes. This phenomenon is widely recognized cascade failure [17].

Watts [18] studied the relationship between average degree of a network and robustness or threshold of each node. The cascade model considers a failure of a node propagate when the fraction of neighboring failed nodes exceeds a threshold of a corresponding node. Let the networked system have $N$ nodes and $M$ links and each node $i$ has the state $s_{i}=0$ (not failed) or $s_{i}=1$ (failed). The state $s$ of nodes depends on the fraction of failed nodes in neighboring nodes. We assume all nodes have same threshold $\phi$ and simultaneously update their state by the following equation.

$$
s_{i}= \begin{cases}1 & \sum_{j \in N_{i}} s_{j} / d_{i} \geq \phi \\ 0 & \sum_{j \in N_{i}} s_{j} / d_{i} \leq \phi\end{cases}
$$

where $s_{j}$ and $N_{i}$ represents the state of node $j$ and adjacent nodes of node $i$ respectively.

The probability that the number of neighbors $d_{i}$ of each node is $k$ follows the degree distribution $\mathrm{P}(\mathrm{k})$. If the $\mathrm{P}(\mathrm{k})$ follows Poisson distribution, almost all nodes have same number of neighbors, while a few nodes have huge number of neighbors if it follows Power law distribution. The degree of node $d_{i}$ also represents the tolerance of node $i$ against external failures. In the case of nodes with small degree, they may be severely impacted by the failure of only single adjacent node, while in the case of hub nodes, they are very stable because they have many other nodes even if one of them becomes failed.

Watts et al. [18] studied the relationship between the average degree $z$ of the network and the threshold $\phi$ against cascading failures. They found that there is a region in which a small amount of failures can make the sequence of node failures and it spreads to the entire network. He defines the region as "cascade window", which can be theoretically derived from the degree distribution $\mathrm{P}(\mathrm{k})$ of the network,

$$
\sum_{k=0}^{K_{*}} k(k-1) P(k)=z, \quad \mathrm{~K}_{*}=\lfloor 1 / \phi\rfloor
$$

He found the cascade failure could occur in the specified area of the average degree and the threshold and this area is named as cascade window as shown in Figure 1. When the network is too sparse (there are few links) to be connected, the impact cannot spread via nodes. When the network is sufficiently dense (there are many links), the size of the impact of failures becomes relatively small because each node has enough number of other nodes to transmit or receive physical quantities. His study about cascade failures implies the important fact that the robustness of each node in the network does not show the robustness of the entire network. A network topology defines where and which nodes communicate or interact with others. In the case of a certain network, a part of failures of a network can spread via vulnerable parts of a network or change the balance of the load drastically. Then, we have to take into account not only the performance of each node but also the network topology to design a robust network to cascade failures.

Gai and Kapadia[19] were the first to analyze the mechanism of systemic risk using a general cascade model of developed by Watts. In their model every bank in the network is assumed to be identical i.e., that all banks have the same numbers of debtors and creditors. They investigated how the system responds to the bankruptcy of a single bank, and in particular when this results in contagion events in which a finite fraction of the banks are shut down. They consider the case of random graphs and homogeneous distribution of assets among banks.

Nier et al.[3] modeled systemic risk in financial networks of heterogeneous banks and find that the more capitalized banks are the more resilient the banking system against contagious defaults is, and this effect is nonlinear. They also modeled a small size of the tiered structure by classifying the banks in the network into large and small banks and find that tiered structures are not necessarily more prone to systemic risk and that whether they are or not depends on the degree of centrality, which is the number of connections to the central node. Such that, as the degree of centrality increases, contagious defaults first increase but then start to decrease, as the number of connections to the central node starts to lead to greater dissipation of the shock.

May and Arinaminpathy[20] provided the analytical approach by their assumption that every bank in the network is identical. This mean-field assumption gives reasonably 
accurate results for random networks, which have independent Poisson distributions for in- and out-degrees. This means that each bank in such a network is similar to the "average" bank. However, real-world banking networks often have fat-tailed degree distributions [10], meaning that there is a significant probability of finding a bank with in-degree (or out-degree) very different to the mean degree.

$\mathrm{Li}$ and $\mathrm{He}$ [21] constructed a tiered network model of the interbank market. From the perspective of contagion risk, they studied numerically the resilience of four types of interbank market network models to shocks, namely, tiered networks, random networks, small-world networks, and scale-free networks. They studied the interbank market with homogeneous and heterogeneous banks and analyzed random shocks and selective shocks. Their study revealed that tiered interbank market networks and random interbank market networks are basically more vulnerable against selective shocks, while small-world interbank market networks and scale-free interbank market networks are generally more vulnerable against random shocks. Besides, the results indicate that, in the four types of interbank market networks, scale-free networks have the highest stability against shocks, while small-world networks are the most vulnerable. When banks are homogeneous, faced with selective shocks, the stability of the tiered interbank market networks is slightly lower than that of random interbank market networks, whereas, in other cases, the stability of the tiered interbank market networks is basically between that of random interbank market networks and that of scale-free interbank market networks.

\section{RESILIENCE OF FINANCIAL}

As the global networks in many fields become increasingly important to all aspects of society, the consequences of disruption become increasingly severe. Thus it is critical to increase the resilience and survivability of these global networks. We define resilience as the ability of the network to provide desired service even when challenged by failures, large-scale disasters, or intentional attacks. We have to develop a comprehensive methodology to evaluate network resilience using a combination of topology generation, analytical, simulation, and experimental emulation techniques with the goal of improving the resilience and survivability of global networks.

The research on networks has developed elaborate mechanisms and strategies to ensure the resilience of critical networking infrastructures. A challenge for researchers is ensuring their output is relevant to practitioners, and can thus lead to the improved resilience of current and future network deployments. This is difficult for several reasons: the challenges of acquiring useful detailed information regarding network outages; the difficulties of wide-scale experimentation with network disruptions such as attacks; the general lack of transparency regarding the challenges faced by network operators and the processes used to mitigate them; and the difficulty of modeling the complex manner in which socio-technical networked systems fail.

We can learn their efforts in order to improve the resilience of global financial networks. In our life, the interconnection of networks, which are relatively small or medium size, is usually used to form a very large-scale network efficiently. In the financial network, city bank, local bank and public-sector financial institutions each has their own network, and they form a huge financial network by their interconnections such as transactional relationship. Furthermore there are other examples in road networks, power transmission networks and social communities. These types of network are called "modular networks", "network of networks" or "community network".

Modularity is an important concept for understanding the structure and the functions of networked systems. A network composed of interconnected sub-networks is called "modular networks", where there are a lot of interactions in each module but a few interactions of inter-modules. Recently, Gao[22] studied the relationship between the number of modular networks and the performance of modular networks connected randomly by using percolation dynamics, which are actually called "interdependent networks". They introduced interdependent links to connect a pair of nodes in different modular networks, which represents the relationship that a function of a node depends on the state of a node connected. The interdependent network is very destructible compared to a single network having only connecting links and the threshold fraction of removed nodes for breaking network connectivity becomes much smaller. Interdependency of modular networks works as an amplifier of the failure of nodes.

Gleeson et al. [4] developed an analytical approach to calculating the expected size of contagion events in networks of a prescribed modular network topology. Their analytical approach gives insights into the mechanisms of contagion transmission in random and scale-free network topologies, and enables formulas relating critical parameter values to be derived. They consider how the extent of the contagion is affected by the size of the bank that initiates the cascade, and so to inform the question of which banks are 'too big to fail'.

Komatsu and Namatame[8] characterized the least and most susceptible network topologies. Such networks are characterized core-periphery structures consists of hub nodes and stub nodes. The hub nodes form a near complete graph, and stub nodes are connected mainly to hub nodes. They consider a modular banking system represented by the scalefree network and study the impact of tiering in such a system on susceptibility to shocks. We need to focus on the effects of variation in the level of tiering, where the level of tiering is represented by the inverse of the degree distribution is such that an absolute majority of banks have very low degree whereas a few banks have high degree. High-degree banks are mainly connected to low-degree ones and those low-degree banks rarely have links anywhere else.

One of the key issues on modular network design is modularized networks are interconnected. Figure 2 shows some features of modular networks as the function of the ratio of links used for linking inter modules. The recursive design model is a network model that interconnects modular networks recursively and we can design a very large-scale network. We propose a recursive network design model as follows: We have a basic unit modular network, P model or CPA (CRA) 
networks which as the largest or smallest cascade window. The overall modular networks have a topology of intermodules, which is the same as a modular network as a basic unit. The formation of the recursive network starts by the creation of a relatively small network as a unit network (modular network). Next $n$ replicas of the unit network are generated, where $n$ represents the number of nodes in the original unit network. Each replicated network is deployed at the position of each node in the unit network, and they are interconnected by links. The proposed design model can deal with modular networks comprising many modules, and it enables the consideration of the influence of the topology of inter-modules toward dynamical processes.

\section{CONCLUSION}

Globally networked risks are difficult to identify, map and understand, since there are often no evident, unique causeeffect relationships. Failure rates may change depending on the random path taken by the system, with the consequence of increasing risks as cascade failures progress, thereby decreasing the capacity of the system to recover. "In certain cases, cascade effects might reach any size, and the damage might be practically unbounded.

Structural features exist on both the microscopic level, resulting from differences between single node properties, the meso-scopic level resulting from properties shared by groups of nodes, and the macroscopic level resulting from properties of the whole nodes such as the degree distribution. We use a meso-scopic approach of contagion models to describe risk propagation in networks and investigate how networktopology impacts risk propagation. Each node fails depending on the states of their neighbors, and systemic risk is measured as the fraction of failed nodes. If each node is connected to other nodes, even if a failure may at some nodes happen relatively frequently all over the network, usually such a failure in one spot is absorbed by neighboring nodes. Such a failure may, however, propagate to other nodes under certain conditions. When that happens, things worsen all along the chain until the network ends up with a widespread failure.

It is know that the interbank loan networks in general evolve over times without any fixed topologies. On the other hand most studies on systemic risks have been done on fixed topologies. In recent studies, the underlying network topology is important for the occurrence of systemic risk,little is known about what is the optimal network topology for minimizing systemic risks. Whether optimization plays a key role in shaping the evolution of optimal networks is an important question. We have studied the robustness of the network against cascade failures based on the threshold model and introduced the most and least susceptible networks to the cascade failures. The network consists of hub nodes and stub nodes that are belong to one of hub nodes in the cluster. One hub node and stub nodes make a module. The network consists of many the modules, and a center cluster (complete graph) of hub nodes unifies them.
Our global networks have generated many benefits and new opportunities. However, they have also established highways for failure propagation.The increasing interdependencies between the world's financial systems have the potential to create global catastrophic risks. We may have to re-design many global networks. The results of modular network design imply that the network topology can drastically reduce the damage of the cascade failures.

\section{ACKNOWLEDGMENT}

The authors would like to appreciate for support by Grantin-Aide for Scientific Research in Japan No. \& No.25330277..

\section{REFERENCES}

[1] A.Haldane, andR. May, "Systemic risk in banking ecosystems."Nature, Vol.469: 351-355, 2011.

[2] P.Gai and S. Kapadia, "Contagion in financial networks." Proc. R. Soc. Interface, 466-480, 2010.

[3] E.Nier, J. Yang, T. Yorulmazer, and A. Alentorn, "Network models andfinancial stability." Journal of Economic Dynamics and Control 31 (6): 2033-2060, 2007.

[4] J.Gleeson, T. Hurd, S. Melnik, and A. Hackett, "Systemic risk in banking networks without Monte Carlo simulation."Advances in Network Analysis and its Applications Mathematics in Industry, Vol.18, pp.27-56, 2013.

[5] S.Battiston et al.,"DebtRank: Too Central to Fail? Financial Networks, the FEDand Systemic Risk."Scientific Reports 2, Nature Publishing, 2012.

[6] M.Rombach,"Core-Periphery Structure in Networks", arXiv:1202.2684[cs.SI], 2013.

[7] Fortunato, S. 2010. "Community detection in graphs." Physics Reports $486(3-5): 75-174$

[8] T.Komatsu and A. Namatame, "Dynamic diffusion processes in evolutionary optimized networks" Int. Journal of Bio-Inspired Computation, Vol.3, 384-392, 2011.

[9] F.Allen and D. Gale, "Financial Contagion." Journal of Political Economy, 108, 1-33, 2000.

[10] M.Boss, H. Elsinger, M. Summe, and S. Thurner,"The Network Topology of the Interbank Market." arXiv:cond-mat/0309582v1, 2003.

[11] B.Craig andG. Peter,"Interbank tiering and money center banks." BIS working paper, 2010.

[12] K.Sorama $\square$ ki, M.L. Bech, J. Arnold, R.J. Glass, and W.E. Beyeler,"The topology of interbank payment flows." Physica A, Vol. 379, pp. 317333, 2007

[13] D.Fricke andT. Lux, "Core-Periphery Structure in the Overnight Money Market.” Kiel Working Paper, 2012.

[14] M.Teteryatnikova, "Resilience of the Interbank Network to Shocks and Optimal Bail-Out Strategy: Advantages of "Tiered" Banking System." Working paper, Dept. of Economics, University of Vienna, 2010.

[15] M.E.J.Newman, “Assortative mixing in networks." Physical Review Letters 89, 208701, 2002.

[16] F.Chungand L. Lu, "The average distances in random graph with given expected degrees." Proc. of the National Academy of Sciences, 99, 15879-15882, 2002.

[17] A.E.Motterand Y.-C. Lai, "Cascade-based attacks on complex networks." Physical Review E, 66 (6):065102, 2002.

[18] D.Watts, “Asimplemodelofglobalcascadesonrandomnetworks." PNAS, 99 (9) 5766-5771, 2002.

[19] P.Gai, A. Haldane, and S. Kapadia, "Complexity, concentration and contagion.”Journal of Monetary Economics, vol. 58, no. 5, pp. 453-470, 2011.

[20] R.May and N. Arinaminpathy,"Systemic risk: the dynamics of model banking system.”J. R. Soc. Interface, Vol.7, pp.823-838, 2010.

[21] S.LiandJ. He, "Resilience of Interbank Market Networks to Shocks." Discrete Dynamics in Nature and Society, Vol. 2011, 121-135, 2011.

[22] J.Gao, S. Buldyrev, E. Stanley, and S. Havlin,"Networks formed from interdependent networks." Nature Physics, Vol.8, 40-48, 2012. 